\title{
Predicting Machining Errors in Turning Using Hybrid Learning
}

\author{
X. Li, P. K. Venuvinod, A. Djorjevich and Z. Liu \\ Department of Manufacturing Engineering and Engineering Management, City University of Hong Kong, Kowloon, Hong Kong
}

\begin{abstract}
A recent model-based approach for predicting the compensation required on the next part to be turned on a CNC machine solely on the basis of three independent measurements conducted at selected locations on a limited set of previously machined parts under a similar cutting set-up is reviewed. A new method of achieving the same objective through the use of the learning capability of an adaptive neuro-fuzzy network is developed and tested against experimental data for cylindrical turning. This method requires only one on-machine measurement per sample. It is conducted by a novel contact sensor that probes with the tool and facilitates automation by providing proximity information as the tool approaches the workpiece.
\end{abstract}

Keywords: Adaptive neuro-fuzzy network; Machining error; Turning

\section{Introduction}

Since CNC machines are expensive, it is important to exploit fully their accuracy capability. However, in industrial practice, the actual machined part dimensions rarely equal the desired (NC programmed) values. For instance, when we tested a CNC turning centre of a well-known brand, we found that the total dimensional error, $\delta_{t o t}$, over the machined part diameter during cylindrical turning operations could reach values as high as $80 \mu \mathrm{m}$, although the positioning error, $\delta_{\text {pos }}$, of each of the machine's axes was of the order of only $\pm 4 \mu \mathrm{m}$ when the machine was in the unloaded and cool state. This means that we are not usually able to exploit fully the accuracy potential of a given machine. The cost of not dealing with this problem can be high because of "unnecessary statistical quality control activities, high scrap rate, cost of rework, late delivery, excessive costs for the slightest tightening of product tolerances, unreliable products in the field and high turnover of people because of discomfort [1]."

Correspondence and offprint requests to: Dr A. Djorjevich, Department of Manufacturing Engineering and Engineering Management, City University of Hong Kong, Tat Chee Avenue, Kowloon, Hong Kong. E-mail:mealex@cityu.edu.hk
Several strategies have been attempted in the past to deal with the problem of machining inaccuracy. These fall into two classes: error avoidance and error compensation. Error avoidance attempts to minimise errors through better machine design, construction, and environmental control via hardware. However, there is a limit to how accurate a machine can be made within an acceptable cost through improved design and fabrication. Error compensation via software aims to anticipate the combined effect of error sources on workpiece accuracy and then modify the conventionally designed tool path. Owing to its reliance on modification at the software level rather than on error avoidance via hardware, error compensation via software has the potential of providing an economical method of achieving accurate machining without accurate machinery.

Although a great deal of research effort has been dedicated to it, error compensation via software is not yet common in industry. A major reason for this is that traditional solutions to the problem have required the collection of large amounts of data using expensive equipment (such as a laser interferometer) on each machine on the shop floor. Sometimes, temperature distribution data are collected from a large number of thermocouples mounted at selected locations on the machine (as many as 100 [1]). An additional impracticality is that the data collected are unique to that machine and are not applicable to other machines even if they are of the same make. Hence, it becomes necessary to outfit each and every machine tool on the shop floor with additional hardware and signal-processing equipment. This can be very expensive and time-consuming. Moreover, most CNC machines routinely encounter workpieces of different materials, shapes, and dimensions. During machining, tools with a variety of tool angles and materials are selected. Likewise, the cutting conditions (cutting speed, feedrate, depth of cut, etc.) can vary over wide ranges. Clearly, it is useful to develop a system that can learn to compensate for machining errors under such variable conditions without the need for expensive additional equipment, i.e. the data needed for enabling error compensation should be obtained essentially from normal shop floor operations. A review of relevant literature indicates that, with the exception of [2,3], there have been few attempts at developing such a system.

In [2,3], an attempt was made to develop an error compensation method that relied solely on post-process and on-machine measurements of parts previously machined on the same 
machine. It was argued that these measurements are routine and normal shop floor activities and, hence, the new approach meets the criterion of shop floor acceptability.

This method was based on the simple premise that any error source is of importance only by virtue of the imprint it leaves on the total error on the part. Hence, it should be possible to recognise the underlying machining-error-related patterns of behaviour of the specific machine tool and the associated machining operations simply from information contained in past part inspection data. Initially, attempts were made to develop an artificial neural network (ANN) based approach that could discern the patterns solely from data consisting of post-process dimensions, $D_{p p}$, as determined by using a coordinate measuring machine (CMM). However, this proved to be cumbersome and impractical. Hence, an attempt was made to "divide and conquer". In particular, two further dimensional measurements complementing $D_{p p}$ were included: an onmachine dimensional measurement, $D_{o m w}$, conducted immediately after completing the machining operation (i.e. while the machine is still warm); and an on-machine measurement, $D_{\text {omc }}$, conducted after the machine had cooled down. It was shown that these three measurements suffice to decompose the total error, $\delta_{t o t}$, into the three major error components (geometric, thermal, and force-induced deflection error components). A simple analytical model was then developed for each of the error components, and the magnitudes of the associated model coefficients were estimated from data on the corresponding error component as obtained from the total error decomposition strategy. However, these magnitudes depended on the specific class of machine set-up, machine thermal loading conditions, cutting conditions, etc. It was proposed that this problem would be solved by using a case-based reasoning (CBR) approach that enabled the magnitude of each error component for the "next" machining operation to be anticipated by retrieving and adapting one or more similar machining operations experienced previously by the machine. If the "next" operation is totally new (i.e. if the machine has not experienced a similar operation previously), the three measurements are repeated on the new operation, and the resulting error component estimates are stored away for future use. Thus, the machine would be able to progressively learn to perform the task of error compensation via software solely on the basis of dimensional measurements conducted on previously machined parts. Extensive data demonstrating the applicability of this approach to cylindrical turning on a CNC turning centre was presented in [2,3].

Another interesting feature of the error compensation strategy explored in [2,3] was the use of a novel on-machine measurement device called the "fine touch" contact sensor that was originally developed at Kiev Polytechnic, Ukraine. The fine touch sensor consists of a coil wound around the tool that produces an electrical signal when the tool contacts the workpiece. Extensive tests have shown that the device is capable of detecting tool-work contact with a repeatability error better than $1 \mu \mathrm{m}$ under varied shop floor conditions [4]. In [5], the automatability of the Fine Touch technique was enhanced by combining it with the Q-setter device that is commonly available on many CNC turning centres. Thus, "fine touch" enables the cutting tool itself to be used as a contact probe during onmachine measurement exercises. This method of on-machine measurement has been found to be more convenient and less expensive than the conventional on-machine measurement method based on the use of a "touch trigger" probe.

Notwithstanding the demonstrated success of the error compensation approach described in [2,3], four difficulties associated with the approach are worth noting:

1. It is difficult to automate the on-machine measurement cycle based on the fine touch technique because this technique requires the tool (the contact probe) to approach the work surface at jogging speed. This slows down the on-machine measurement process substantially.

2. The approach requires three independent measurements ( $D_{o m w}, D_{o m c}$, and $D_{p p}$ - in that order) to be made with respect to each part dimension on which data must be collected. This can be tedious and time consuming.

3. The error compensation strategy uses a model-based approach where a separate model must be developed for each major error component in the context of each cutting situation class. While this method has worked well in the context of cylindrical turning of workpieces held in a chuck at one end with the other end free [2,3], it may not be possible to develop simple analytical models for other cutting situations such as workpieces supported additionally by a tail stock, end milling, etc.

4. Learning through case-based reasoning is, by nature, episodic and, therefore, intermittent. Further, learning takes place on the basis of information contained only on similar retrieved cases. Information embedded in other cases is not used. These features could slow down the learning rate substantially.

The present paper aims to develop a modified error compensation strategy that addresses at least the first three of these four problems.

The paper is organised as follows. First, a new contact sensing technique, called "soft touch", will be described. This technique represents an improvement over the fine touch technique used in [2-4]. The new technique is more easily automated since it can detect tool-work proximity in addition to tool-work contact. Next, the error decomposition strategy developed in [2,3] will be reviewed briefly with a view to reducing the number of dimensional measurements needed from three to one. This will be followed by a description of a neuro-fuzzy technique and its use in predicting the combined thermal and force-induced deflection error. Next, experimental data based on this simplified measurement strategy will be presented and the effectiveness of the neuro-fuzzy system demonstrated on the basis of these data. Finally, the shop floor level applicability of the new system will be discussed critically so as to identify areas requiring further work.

\section{The "Soft Touch" Sensor and its Application in Error Measurement}

On-machine inspection provides a real-time insight into the machining process. Traditionally, such inspection has been performed using "touch trigger" (TT) contact probes. Notwith- 
standing their widespread use in industry today, there are two major problems with these probes. First, they are expensive and delicate. Secondly, the current cutting tool must be replaced by the TT probe each time an on-machine cycle is initiated.

An interesting solution to both problems has been provided recently by the "fine touch" (FT) probing strategy described in Section 1. The FT technique enables the cutting tool itself to be used for the detection of tool-work contact. When the FT probe is used in combination with the Q-setter available on many CNC turning centres, the tool-work contact position coordinates can be read from the axis control system of the machine itself [5]. This eliminates the need:

1. For an expensive and delicate device like a TT probe.

2. For exchanging the cutting tool with a separate probe.

Further, because the probe is the cutting tool itself, the inaccessibility problem associated with other direct measurement methods is eliminated [6,7] (in applications where tool wear is significant and the tool offset changes, a periodic recalibration may be carried out on-machine [7]).

A problem with the FT technique is that it can detect toolwork contact but not proximity. Hence, a large allowance must be made while determining the axis position, where the tool motion has to be changed from rapid to slow (jogging) motion as the tool approaches the work surface for the purpose of contact detection. This problem would not exist if we could extract tool-work proximity information in real-time. We have recently developed a new technique, called "soft touch" (ST) to solve this problem.

Unlike the FT technique, our ST technique provides proximity information as the tool approaches the workpiece, in addition to the binary on/off signal indicating contact/no-contact. The main component of the sensor is a coil of wire placed around the tool holder that acts as the coil's ferrite core (Fig. 1). Together with a small capacitor, this coil forms an oscillator whose natural frequency is a function of the variable coil inductance (assuming other parameters are kept constant). Background electromagnetic (EM) radiation and ground loops through the machine provide the oscillator excitation. A load resistor followed by an analogue amplification stage (an Op-amp) completes the sensor electronics. We monitor the frequency shift in the power spectrum of the amplified oscil-

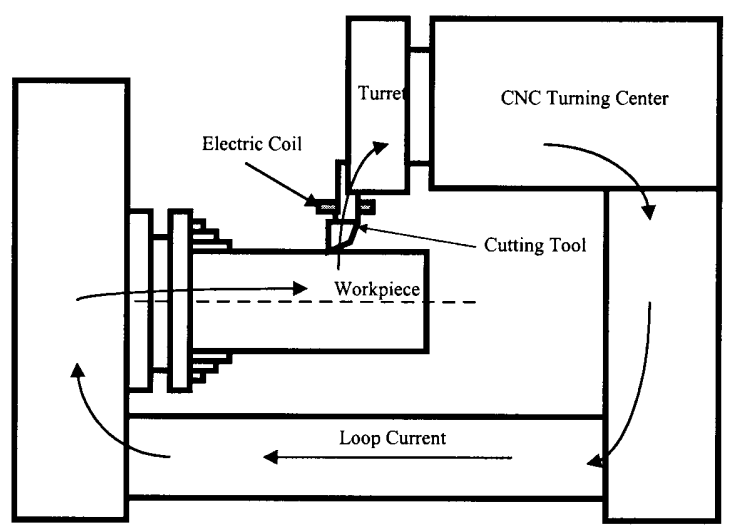

Fig. 1. Fine/soft touch tool-work contact sensor as used in turning. lator signal. Its gradual shift corresponds to the tool-approaching phase in the micrometer range and is useful as a warning of the impending tool contact with the workpiece. The latter event causes a much more drastic frequency shift that is better characterised as a jump.

The sensor behaves like an oscillator, transmitting the background EM noise from ambient sources (motors, transformers, etc.) that was guided along the tool holder, provided that the frequency of such EM excitation is around the natural (resonant) frequency of our transmitter/oscillator. In other words, of all the excitation frequencies, only those around the oscillator's natural (resonant) frequency will attain magnitudes strong enough to be transmitted, most others are attenuated.

The oscillator's natural frequency depends on the coil inductance. The latter is affected by the magnetic coupling (state of contact) between the tool and workpiece as the two components of the coil's core. We therefore monitor the state of the tool-to-workpiece proximity/contact by monitoring the natural frequency of the oscillator, which is achieved by observing what noise frequencies are being transmitted.

Numerous tests against a laser interferometer showed that the contact detection accuracy of the instrument itself is better than $1 \mu \mathrm{m}$. However, because the absolute sensor position at the time of contact is read on the machine's tool position coordinate display, the overall measurement accuracy is governed by the accuracy of the machine's motion axes, often in the micrometer range.

It is stressed that we monitor for the sudden frequency shift (the derivative of the shift) in the power spectrum, and not the actual value of that frequency. This allows sensor application to be used over a variety of tool and workpiece material combinations, not necessarily ferritic.

The frequency shift typically seen by the fine touch sensor during the final $2 \mu \mathrm{m}$ motion of the tool is shown at the upper part of Fig. 2. The rising edge of the second, sharper hump represents tool contact and is followed by its plunging into the workpiece. The ripples observed probably represent the sticking motion of the machine axis. The lower part of Fig. 2 shows the receiver-signal output (voltage) magnitude. It provides an alternative means of capturing the instant of the tool's contact with the workpiece. This voltage variation is believed to be the consequence of the oscillator's resonant frequency shift towards or away from some dominant frequency in the

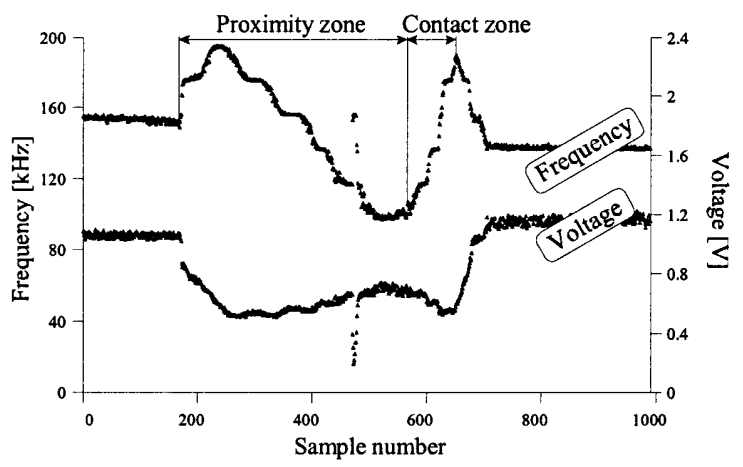

Fig. 2. Either the magnitude or frequency shift of the soft-touch sensor signal may be monitored. (At the scale presented, lengths distorted by motion velocity: proximity zone appears compressed.) 
background EM noise (such as a power-supply frequencymultiple). As the resonant frequency shifts closer to or away from this excitation frequency, a higher or lower magnitude, respectively, is detected on the receiver. Although such sensor use is simpler to implement since it does not require power spectrum analysis, voltage drift poses a problem over long periods, giving preference to signal processing in the frequency domain. The error decomposition approach described in the next section is not influenced by which of the two alternatives is chosen.

\section{Dimensional Error Decomposition}

Suppose $D_{\text {des }}$ is the desired (NC programmed) part diameter in a cylindrical turning operation. Then, the actual diametral error, $\delta_{t o t}$, after the part has been machined can be assessed as Dpp by performing a post-process inspection operation (e.g. using a CMM). Then, clearly,

$$
D_{p p}-D_{d e s}=\delta_{t o t}
$$

On the other hand, $\delta_{t o t}$ is the algebraic sum of quasi-static errors arising from several sources and can be expressed for the case of cylindrical turning as follows:

$$
\delta_{t o t}=\delta_{g}+\delta_{t h}+\delta_{f}+\delta_{\text {other }}
$$

where,

$\delta_{g} \quad$ is the diametral error component arising from the geometric errors associated with the axis motions

$\delta_{t h} \quad$ is the diametral error component arising from the thermally induced (induced by temperature variations and gradients within the machine structure) toolwork displacements

$\delta_{f} \quad$ is the diametral error arising from tool-work displacement resulting from the overall deflection pattern of the machine-fixture-workpiece-tool (MFWT) system of the machine under the action of the quasi-static cutting force

$\delta_{\text {other }}$ is the summation of other diametral error components such as those arising from clamping forces, tool wear, etc.

Irrespective of the probe used (touch trigger, fine touch, or soft touch), let $D_{o m}$ be the dimension corresponding to $D_{p p}$ as determined by on-machine measurement. Mou and Liu [8] have demonstrated (empirically) that

$$
D_{p p}-D_{o m}=\delta_{p o s}
$$

where $\delta_{\text {pos }}$ is the positioning error of the machine.

The rationale behind Eq. (3) can be explained as follows. Suppose we start with a perfectly cylindrical workpiece whose diameter, $D_{p p}$, we will measure on a CMM. We now mount this perfectly cylindrical part on our turning centre and conduct an on-machine measurement. The on-machine measurement $D_{o m}$ will not be equal to $D_{p p}$ because our machine tool, unlike the CMM that may be assumed to be perfect for all practical purposes, has significant positioning error $\delta_{\text {pos }}$. In fact, the onmachine measurement operation will detect this positioning error. This explains Eq. (3).
Consider now the significance of the timing of on-machine measurement. Suppose that we had conducted the measurement immediately after the cutting operation, i.e. while the machine was still in the same thermal state as at the time of cutting. Suppose the magnitude of $D_{o m}$ thus obtained was equal to $D_{\text {omw }}$ (the additional suffix " $w$ " indicates that the machine was in the "warm" state). Note that this measurement was obtained with the same positioning error as that which had existed during machining. Clearly, this positioning error in this state would be equal to $\left(\delta_{g}+\delta_{t h}\right)$. Hence, following Eq. (3), we have

$$
D_{p p}-D_{o m w}=\delta_{g}+\delta_{t h}
$$

So that, combining Eqs (1), (2) and (4), it follows that

$$
\delta_{f}=\delta_{\text {other }}=D_{o m w}-D_{\text {des }}
$$

Consider now the significance of $D_{\text {omc }}$, the dimension found through on-machine measurement conducted after the machine has completely cooled down, i.e. after the machine's thermal errors have disappeared (the machine is "cool"). Hence, the measurement is being conducted with the positioning error equal to $\left(\delta_{g}+\delta_{\text {other }}\right)$ so that

$$
D_{p p}-D_{o m c}=\delta_{g}
$$

From Eqs (1), (2) and (4), it follows that

$$
\delta_{\text {th }}+\delta_{f}+\delta_{\text {other }}=D_{\text {omc }}-D_{\text {des }}
$$

Figure 3 illustrates the above relationships between machining error components and inspection data in a graphical manner. It is clear that the magnitudes of the individual error components can be determined unambiguously from two on-machine measurements $\left(D_{o m w}\right.$ and $D_{o m c}$, in that order) followed by one post-process measurement $\left(D_{p p}\right.$ provided that the magnitude of $\delta_{\text {other }}$ is negligible. Indeed, this was the procedure followed in $[2,3]$ with a view to achieving total error decomposition. In that work, the magnitudes of the coefficients involved in the analytical model for each error component were determined from data collected with respect to the particular error component. These coefficients were then used to predict the total error on the "next" part to be machined. However, notwithstanding the demonstrated success of the model-based approach, it is important to recognise that it suffers from several problems (four specific problems have been highlighted in Section 1). In the next section, we will discuss an alternative neural netbased strategy that can resolve these problems.

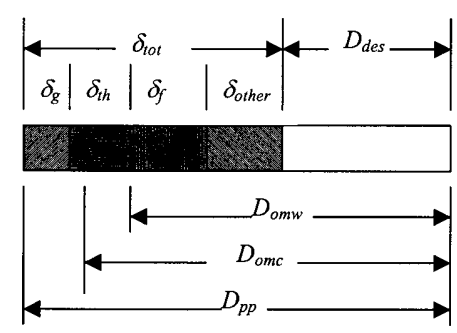

Fig. 3. The relationships between error components and inspected part dimensions. 


\section{Synergy Between Modelling and Learning Through the Use of an Artificial Neural Net for the Purpose of Machining Error Prediction}

A recent review [9] has indicated that the present state of machining process modelling cannot provide reliable solutions to most machining problems. A need was suggested [9] for augmenting process modelling with sensing and learning (through ANN, etc.). In such synergy, modelling would capture a deep understanding of the portions of the process for which the cause-effect relationships have been described analytically. In contrast, a neural network would act as a black box capable of capturing the patterns implicit in other input-output combinations presented to it. This paper follows such an outline in solving the present problem of machining accuracy by applying an ANN-based approach only to that part of the problem that is too complex to be modelled reliably.

According to Eq. (2), the actual dimension error $\delta_{t o t}$ consists of four components: $\delta_{\text {other }}, \delta_{g}, \delta_{t h}$, and $\delta_{f}$. Factors affecting the magnitudes of these error components are complex. Many are not amenable to reliable modelling. Hence, we may use a suitable ANN to take into account the influence of such factors on machining accuracy. However, at least two factors seem to be amenable to predictive modelling: geometric error and cutting forces.

Several successful approaches to the modelling of geometric error $\left(\delta_{g}\right)$ distribution across the workspace of most typical machine tools have already been established (the associated references are too numerous to be cited here) [10]. Further, the magnitude of $\delta_{g}$ usually remains quite stable over time it changes mainly because of slow machine wear or the occasional abuse of the machine. Hence, it is adequate if the distribution of $\delta_{g}$ is measured periodically (e.g. semi-annually). It is unwarranted to use a neural net to identify the patterns underlying this distribution.

A review of machining literature reveals several reliable models for predicting the magnitudes of the three cutting force components (which, in the context of cylindrical turning, are $F_{t}$ tangential to the workpiece, $F_{t}$ : directed along the workpiece radius, and $F_{a}$ : directed along the workpiece axis) arising in several practical machining operations (including the turning operation) [11,12]. For instance, Liu [3] has recently demonstrated good correlation between the force magnitudes predicted through the application of a single-edge oblique cutting model developed recently by Venuvinod and Jin [13] to a set of cylindrical turning operations performed on mild steel and aluminium alloy workpieces. Figure 4 shows how well the predicted radial force, $F_{r}$, had correlated with the corresponding actual force component measured by a three-component piezoelectric cutting force dynamometer. Note that the slope of the regression line (0.959) is close to 1 , the intercept of the regression line (smaller than $4 N$ ) is close to 0 and the coefficient of correlation $(r=0.971)$ is quite close to 1 . Similarly, encouraging results were obtained with respect to $F_{t}$ and $F_{a}$ : the regression line slopes were 0.981 and 0.972 , respectively, the intercepts were $0.9 \mathrm{~N}$ and $2.3 \mathrm{~N}$, respectively, and the correlation coefficients were 0.995 and 0.989 , respectively.

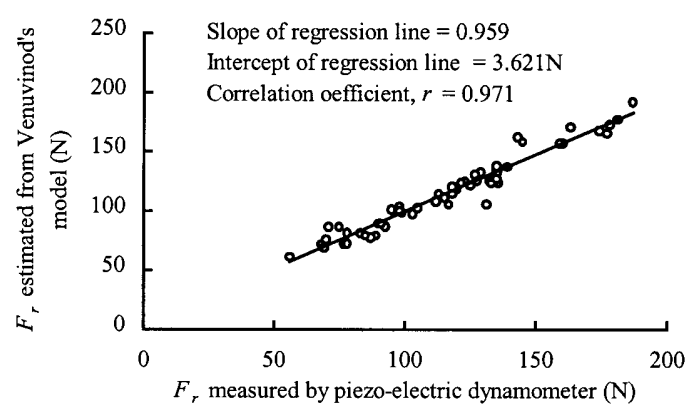

Fig. 4. Effectiveness of the cutting force model[13] in predicting the radial force, $F_{r}$, in turning.

It should be noted that the cutting force magnitudes affect $\delta_{f}$. Likewise, they influence the cutting temperature and internal thermal effects (thus influencing $\delta_{t h}$ ) and tool wear (a factor directly affecting $\delta_{\text {other }}$ ). Hence, it is useful to examine whether the machining error excluding $\delta_{g}$ (i.e. only $\delta_{t h}, \delta_{f}$ and $\delta_{\text {other }}$ ) could be predicted effectively by a neural net whose inputs include the three force components $\left(F_{t}, F_{a}\right.$, and $\left.F_{r}\right)$. In fact, this is the objective of the work reported here.

Now consider the desirability of using a separate neural net for learning the behaviour patterns of $\delta_{t h}, \delta_{f}$, and $\delta_{\text {other }}$ individually and then aggregating the results; as opposed to using a single neural net to learn the aggregated behaviour pattern. We believe that the latter (aggregated) approach is preferable because there is much in common in the input parameters affecting $\delta_{o t h e r}, \delta_{t h}$, and $\delta_{f}$. For instance, the very same parameters (tool/work properties, tool geometry parameters and cutting conditions significantly affect tool wear (a major contributor to $\delta_{\text {other }}$ ). Moreover, it follows from Eq. (7) that the desired aggregate estimate of errors $\delta_{t h}, \delta_{f}$, and $\delta_{\text {other }}$ (that are difficult to estimate through modelling) can be achieved simply through $D_{\text {omc }}$. Therefore, only one on-machine measurement is required, after the machine has cooled down. This eliminates the making of three independent measurements that the modelbased approach [2,3] had entailed. In the rest of the paper, we shall represent the algebraic summation of $\delta_{t h}, \delta_{f}$, and $\delta_{\text {other }}$ by the label " $\delta_{a g g}$ ".

It is fortunate that Eq. (7) contains $D_{\text {omc }}$ rather than $D_{\text {omw }}$ since the use of $D_{\text {omw }}$ would have required the on-machine measurement to be conducted immediately after completing the cutting operation. The greater the time lag between cutting and measurement, the greater the error in implementing Eq. (7). In contrast, no such problem exists with the use of $D_{\text {omc }}$.

Finally, it is useful to note that the force-induced deflection error, $\delta_{f}$, of the MFWT system can be written as

$$
\delta_{f}=\delta_{f, w}+\delta_{f, m f t}
$$

where $\delta_{f, w}$ is the contribution to $\delta_{f}$ due to workpiece deflection on its own under the action of the cutting force and $\delta_{f, m f t}$, is that due to the deflection pattern of the machine-fixturetool assembly.

Liu [3] has investigated the individual distributions of $\delta_{f, w}$ and $\delta_{f, m f t}$, along the workpiece length for the specific case of CNC turning of workpieces held in a chuck at one end with the other end left free. He noted that the distribution of $\delta_{f, m f t}$ is essentially linear, which could be explained by assuming 
that it is caused by a rotation of the workpiece around a "centre of rotation" located somewhere behind the chuck. However, the distribution of $\delta_{f, w}$ will, in general, be nonlinear. In particular, the distribution can be expressed in a cubic form in situations when the workpiece could be idealised as a cantilever of uniform section. However, the analysis will be more complex when the work section is non-uniform (as in the case of a profiled workpiece with or without internal holes). Liu [3] has developed a finite difference program that is capable of predicting $\delta_{f, w}$ with an error smaller than $2 \%$, simply from the knowledge contained in the CAD file of the workpiece and the magnitude of the modulus of elasticity of the work material, even in cases when the workpiece cross-section is highly non-uniform. This suggests that it is not necessary to burden whatever ANN-based learning strategy we intend to use with the task of predicting $\delta_{f, w}$. Therefore, in general, it suffices to "learn" the pattern of $\left(\delta_{t h}+\delta_{f}+\delta_{\text {other }}-\delta_{f, w}=\right.$ $D_{\text {omc }}-D_{\text {des }}-\delta_{f, w}$ ).

This is not the first time that the use of neural nets for the study of machining errors has been attempted. Examples of the application of back-propagation nets and wavelet nets for solving the problem of machining accuracy include [14] and [15]. However, our approach differs in the proposal to use $D_{\text {omc }}$ and fine touch/soft touch as the keys. Further, as will be clear from the next section, our approach also differs in the type of neural net used.

\section{Adaptive Neuro-Fuzzy Inference System (ANFIS)}

Neural networks are said to be low-level computational structures that can offer good performance in dealing with sensory data. In comparison, fuzzy logic can provide higher-level reasoning; however, it has limited learning capability. It would be difficult for a human operator to tune fuzzy rules and membership functions from the training data set. Hence, a promising approach is to fuse fuzzy systems and neural networks into a single system that would combine the benefits of the two $[16,17]$. The resulting neuro-fuzzy system - a hybrid has fuzzy system architecture, but uses a neural learning technique so that it can be trained automatically. The work reported in the present paper uses an example of such a system: the adaptive neuro-fuzzy inference system (ANFIS) [17]. For a given input/output data set, ANFIS can construct a fuzzy inference system whose membership functions are tuned using either a back-propagation algorithm alone, or in combination with the well-known least-squares method.

ANFIS consists of five layers (see Fig. 5). Every node $i$ in Layer 1 is assigned an adaptive fuzzy function in the form of a generalised bell curve:

$$
\mu_{A}(x)=\frac{1}{1+\left|\frac{x-c_{i}}{a_{i}}\right|^{2 b_{i}}}
$$

where $x$ is the node input, $A$ is a label associated with this node and $\left\{a_{i}, b_{i}, c_{i}\right\}$ is a parameter set. By adjusting these parameters, the bell-shaped curve; and therefore the corresponding membership function, can be made to take different forms.

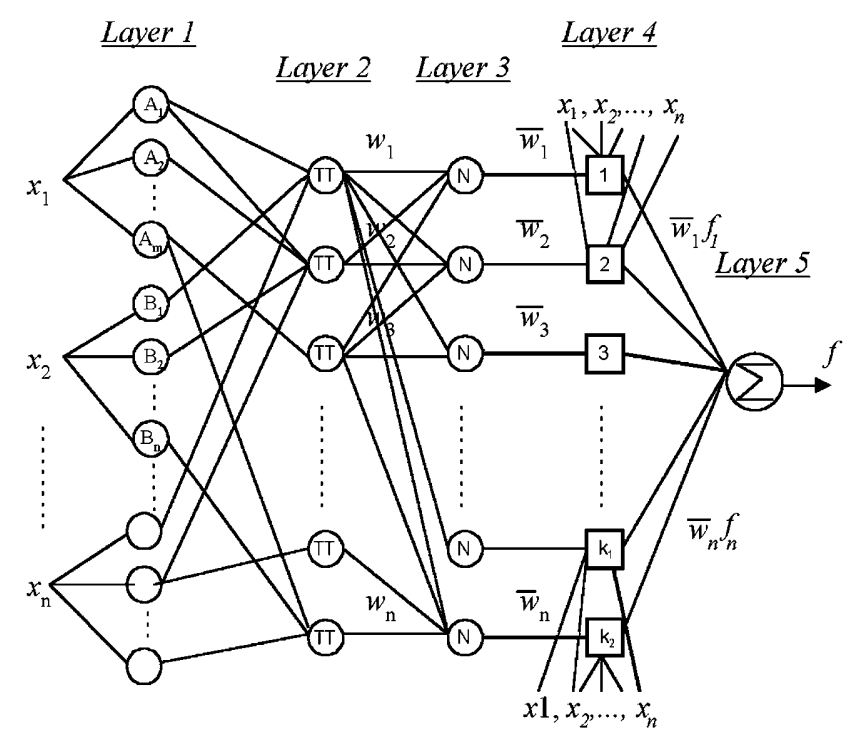

Fig. 5. ANFIS with five layers.

The output of each node in the first layer is a membership grade specifying the degree to which a given input $x$ satisfies the qualifier (or rule) $A$. Every node in layer 2 (labelled $\Pi$ ) is fixed, with its output being a product of all the incoming signals. These node outputs represent the firing strengths of the corresponding rules. Nodes in layer 3 are also fixed (labelled $N$ ). Each one calculates the ratio of the corresponding rule's firing strength to the sum of strength of all rules. Every node $i$ in layer 4 is an adaptive node with the following node function:

$$
\bar{w}_{i} f_{i}=\bar{w}_{i}\left(p_{i, 1} x_{1}+p_{i, 2} x_{2}+\ldots+p_{i, n} x_{n}+r_{i}\right)
$$

where $\bar{w}_{i}$ is the normalised firing strength from layer 3 and $\left\{p_{i j}, r_{i}\right\}$ is the parameter set of this node. The signal node in layer 5 is a fixed node (labelled $\Sigma$ ), which computes the overall output as the sum of the incoming signals:

$$
f=\sum_{i} w_{i} f_{i}=\frac{\sum_{i} w_{i} f_{i}}{\sum_{i} w_{i}}
$$

Parameter sets $\left\{a_{i}, b_{i}, c_{i}\right\}$ and $\left\{p_{i j}, r_{i}\right\}$ are referred to as the premise and the consequent parameter sets, respectively. These are determined through hybrid learning. Since $p_{i j}$ and $r_{i}$ are coupled linearly, these can be identified by the linear least-squares method. Parameters $a_{i}, b_{i}$, and $c_{i}$ are identified by the steepest descent method. We have chosen this hybrid-learning algorithm because it is known to be able to identify its parameters quickly [17].

\section{Experimental Data Used for Training and Testing the Neuro-Fuzzy System}

The experimental data used while implementing the neural network approach was essentially a subset of the data reported in $[2,3]$. The machine used was a 2-axis horizontal CNC turning centre with a six-tool turret. Before performing the machining tests, the geometric error $\left(\delta_{g}\right)$ distribution across the used workspace of the machine was assessed using a laser 
$\mathrm{Z}$ coordinate $(\mathrm{mm})$ $\begin{array}{lllllllllll}0 & 20 & 40 & 60 & 80 & 100 & 120 & 140 & 160 & 180 & 200\end{array}$

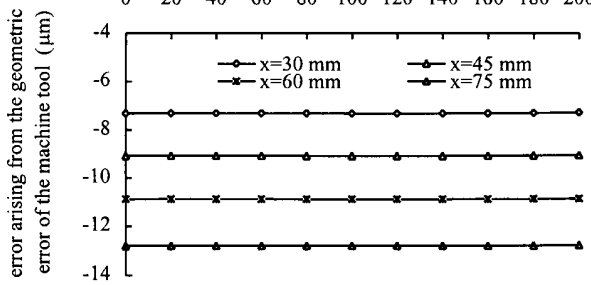

Fig. 6. Distribution of $\delta_{g}$ as determined by a laser interferometer.

interferometer. Figure 6 shows the results. These distributions showed little variation when the tests were repeated several months later. Note that $\delta_{g}$ is sensitive mainly to the radial direction (along the $X$-axis of the machine whereas it remains essentially constant along the axial direction ( $Z$-axis). These observations confirmed the validity of the suggestion made in the previous section that it is unwarranted to load the neural net with the task of figuring out the patterns of $\delta_{g}$.

The experimental data consisted of those obtained from single-point cylindrical turning operations performed on mild steel and aluminium alloy workpieces using DNMG 1506-04QM and 1506-08-QM tools mounted on a piezo-electric threecomponent dynamometer with the fine touch sensor in place as shown in Fig. 1. The sensed information extracted for each cylindrical turning operation consisted of the three measured cutting force components $\left(F_{t}, F_{r}\right.$, and $\left.F_{a}\right)$ along with the diameter, $D$, of the workpiece. After each turning operation, on-machine inspection using a fine touch sensor was performed at selected locations along the workpiece axis after the machine had been allowed to cool down so that the distribution of the $\delta_{\text {agg }}\left(=\delta_{t h}+\delta_{f}+\delta_{\text {other }}\right.$, see Eq. (7)) could be determined.

In order to reduce the number of tests that the evaluation of effects of one factor at a time would have implied, Taguchi's orthogonal arrays were applied to select the cutting conditions for the training and testing data sets. The following settings for cutting conditions were used: cutting speed, $V$, two levels (2.5 and $\left.4.0 \mathrm{~m} \mathrm{~s}^{-1}\right)$; feedrate, $f$, three levels $(0.1,0.15$, and $\left.2.0 \mathrm{~mm} \mathrm{rev}^{-1}\right)$; depth of cut, $d$, two levels $(0.5$ and $1.0 \mathrm{~mm})$; work diameter, $D,(30,40$, and $50 \mathrm{~mm})$; tool nose radius, two levels $(0.4$ and $0.8 \mathrm{~mm})$; and coolant states, two levels (with and without coolant). This procedure resulted in 9 sets of training data obtained while machining mild steel and 11 sets obtained while machining aluminium alloy. Likewise, the data used to test the effectiveness of the ANFIS consisted of 6 additional sets of data (see Table 1 for details of cutting

Table 1. Cutting conditions associated with the test cases.

\begin{tabular}{lllllll}
\hline $\begin{array}{l}V \\
\left(\mathrm{~m} \mathrm{~s}^{-1}\right)\end{array}$ & $\begin{array}{l}f \\
\left(\mathrm{~mm} \mathrm{rev}^{-1}\right)\end{array}$ & $\begin{array}{l}d \\
(\mathrm{~mm})\end{array}$ & $\begin{array}{l}D \\
(\mathrm{~mm})\end{array}$ & $\begin{array}{l}\text { Nose } \\
\text { radius } \\
(\mathrm{mm})\end{array}$ & Coolant & $\begin{array}{l}\text { Work } \\
\text { material }\end{array}$ \\
\hline 2.50 & 0.10 & 1.00 & 45 & 0.4 & Yes & Steel \\
4.00 & 0.20 & 1.00 & 35 & 0.8 & No & Steel \\
4.00 & 0.15 & 1.00 & 45 & 0.8 & Yes & Steel \\
4.00 & 0.15 & 0.50 & 35 & 0.4 & No & A1 \\
2.50 & 0.15 & 1.00 & 45 & 0.4 & Yes & A1 \\
4.00 & 0.10 & 0.50 & 35 & 0.8 & No & A1 \\
\hline
\end{tabular}

conditions). Note that these data include two levels each of $V$, $f, d, D$, tool nose radius, coolant state, and work material.

\section{Performance of the Neuro-Fuzzy Strategy in Predicting $\boldsymbol{\delta}_{\text {agg }}$ Distributions}

Data points labelled $(*)$ in Fig. 7 show the $\delta_{a g g}$ values determined through on-machine measurements, $D_{\text {omc }}$, conducted at 10 positions, $10 \mathrm{~mm}$ apart, for each of the 6 test cases given in Table 1. Note that the distribution of $\delta_{a g g}$ along the workpiece length is nonlinear (it was explained in Section 4 that this arises essentially owing to workpiece deflection on its own). Similar nonlinear distributions were observed in the 20 training sets of data.

Notwithstanding the nonlinear $\delta_{a g g}$-distributions described above, we linearised each of the deviation curves so that each $\delta_{\text {agg }}$ distribution could be expressed as

$$
\delta_{a g g}=a L+b
$$

where $L$ is the axial distance of the point of interest from the face of the chuck, and $a$ and $b$ are appropriate constants.

The main motivation behind this arbitrary simplification was the desire to present a simple input array to the neural net so as to accelerate the learning process. Further, the experimental data used in testing the ANFIS have been derived mainly from turning experiments conducted on relatively short workpieces. This meant that the errors associated with the linearity assumption are likely to be small. In fact, we will show below that, despite the linearity assumption, ANFIS is able to predict quite successfully in the specific context of the data used. Finally,
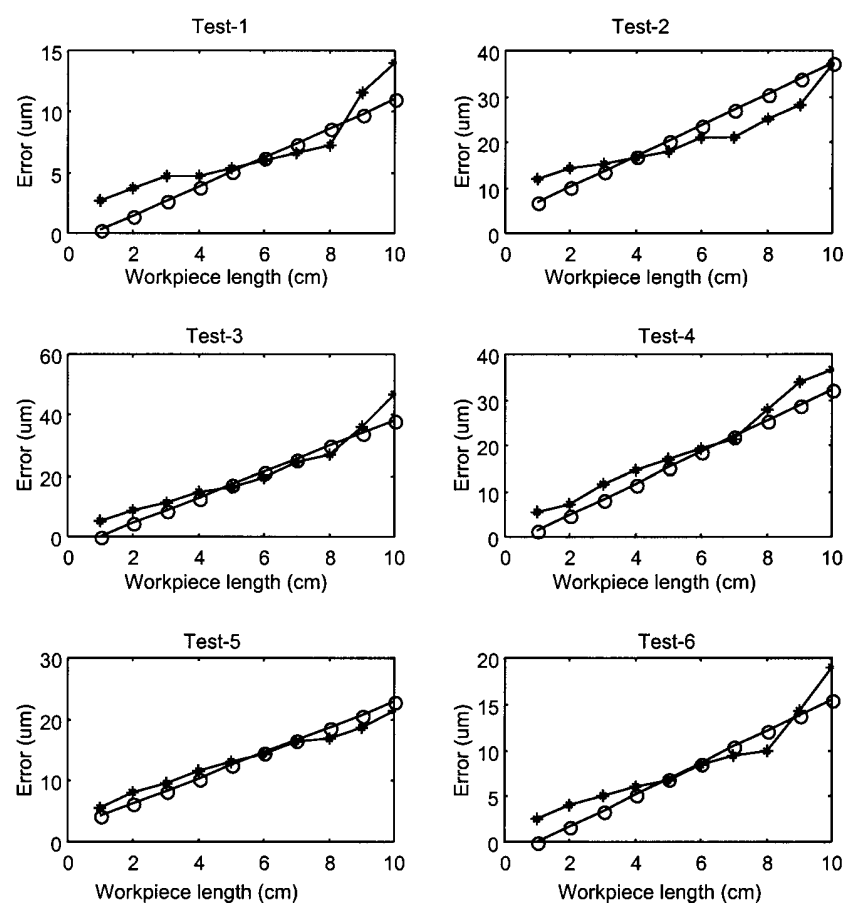

Fig. 7. *, Actual and $\bigcirc$, predicted variations of $\delta_{a g g}$ along workpiece length. 
(a)

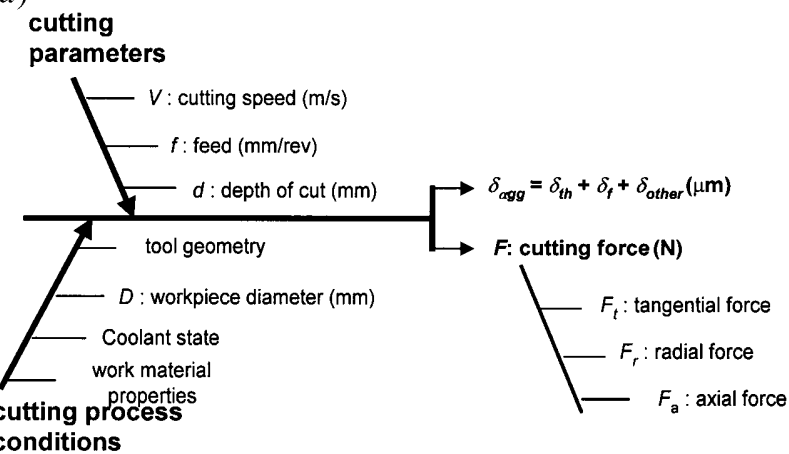

(b)

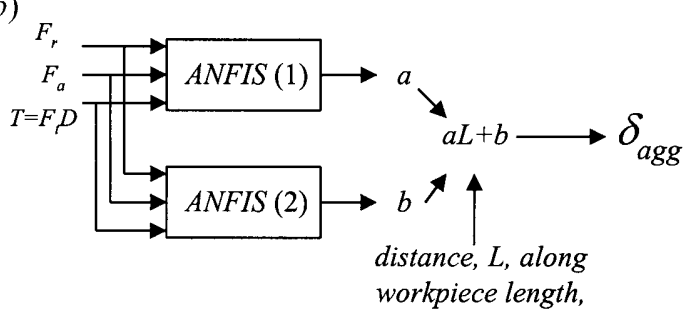

Fig. 8. Mapping between input and output achieved by the ANFIS.

as explained in Section 4, the linearity assumption can still be applied quite reliably, even in the case of long workpieces, provided that (i) we estimate $\delta_{f, w}$ on the basis of the information contained in the CAD-file of the workpiece, and (ii) subtract the resulting estimate of $\delta_{a g g}$ from $\delta_{f, w}$ before training ANFIS to learn the latter.

Next, with a view to reducing the number of inputs to ANFIS, we combined the tangential force component, $F_{t}$, and the part diameter $D$ into a single variable so as to yield the spindle torque, $T: T=F_{t}(D / 2)$. Thus, the array $\left\{T, F_{r}, F_{a}\right\}$ was used as the input to the ANFIS. The outputs from the ANFIS were parameters $a$ and $b$ required in Eq. 11. Figure 8 shows how ANFIS mapped the relationship between its three inputs and two outputs.

ANFIS-2 for parameter $b$, for example, had 78 nodes, 27 fuzzy rules and 108 linear and 27 nonlinear parameters (135 in total), applied on 83 training data pairs. Three bell-type membership functions were used while aiming for the training error of 0.01 (goal), with the initial step size of 0.5 (step size decrease of 0.9 , and increase rate of 1.2). The training phase completed at epoch 1000, with the minimal training RMSE (root mean square error) of 0.108657 (see Fig. 9).
With the $x$-axis expressed as a percentage of a selected nominal case, Fig. 10 shows the effects of parameter learning on the membership functions (MF) of the input features. Their mean values and variances were adjusted during training to minimise the network error.

For parameters $a$ and $b$, Fig. 11 shows the correlation between the values predicted by ANFIS and the corresponding actual values recorded for test sets 1 to 6 . For the training data, the actual values of these parameters were obtained through linear regression. The finally predicted $\delta_{a g g}$ distributions for the 6 test cases in Table 1 are shown by data points labelled $(\bigcirc)$ in Fig. 7. Some statistics related to the error prediction are given in Table 2. Note that the degree of error is quite small (of the order of the positioning repeatability of $\pm 4 \mu \mathrm{m}$ of the machine's axes), thus indicating that the ANFIS strategy had succeeded to a fair degree.

\section{Discussion and Conclusions}

The results presented indicate that the neuro-fuzzy strategy attempted in the present work has succeeded fairly well in predicting the aggregate error $\left(\delta_{a g g}\right)$, i.e. we have been able to predict the algebraic sum of the thermal error $\left(\delta_{t h}\right)$, forceinduced MFWT deflection error $\left(\delta_{f}\right)$, and the further error $\left(\delta_{\text {other }}\right)$ arising from other sources such as tool wear in cylindrical turning operations. This aggregate error may be algebraically added to the geometric error $\left(\delta_{g}\right)$ so as to determine the total dimensional error $\left(\delta_{t o t}\right)$. This total error can then be used to compensate for machining errors following the parametric CNC programming approach described in $[2,3]$.

The main advantage of the new adaptive neuro-fuzzy system (over the model-based approach [2,3]) is that it requires only one on-machine measurement (rather than three) on previously machined parts. This measurement can now be automated because the purposely developed "soft touch" contact sensor with the tool itself as the probe provides the proximity information as the tool tip approaches the work surface.

The new neuro-fuzzy approach has avoided the need for modelling the tool-work displacement arising from the compliance of the MFWT system. This is an advantage since a CNC machine usually encounters a variety of work-holding set-ups during its shop-floor experience, some of which are not amenable to simple modelling. However, the new neurofuzzy approach still requires the model-based prediction of the cutting forces. This synergy of modelling and learning is (a)

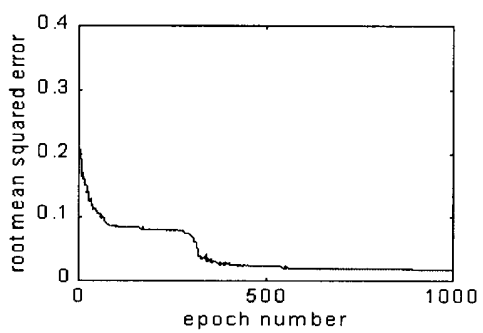

(b)

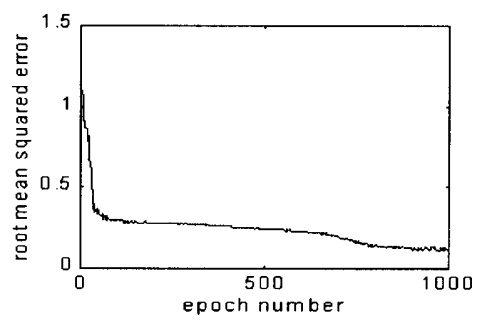

Fig. 9. Decreasing training error of ANFIS with increasing epoch number. Error curves, $(a)$ parameter $a$, $(b)$ parameter $b$. 
(a)

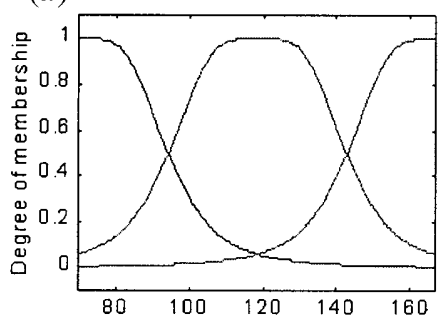

(c)

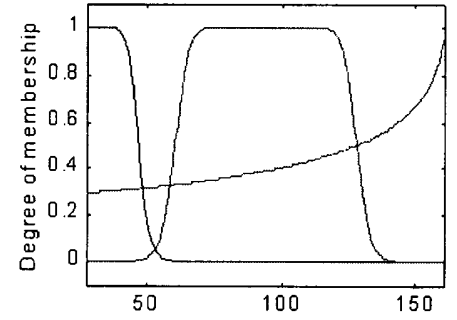

(b)

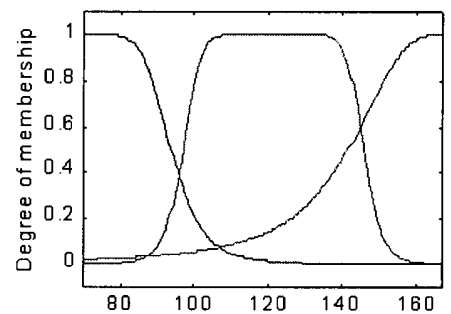

(d)

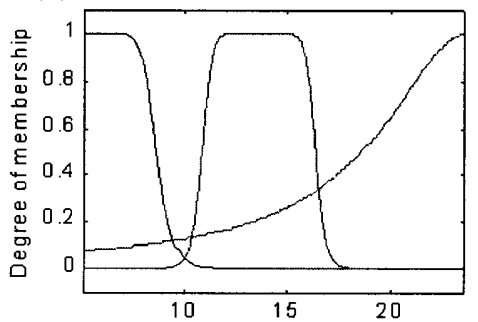

Fig. 10. Learning the membership functions (MF). (a) Initial MF for $F_{r}, F_{a}$, and $T$. (b) Final MF for $F_{r} .(c)$ Final MF for $F_{a}$. (d) Final MF for $T$.
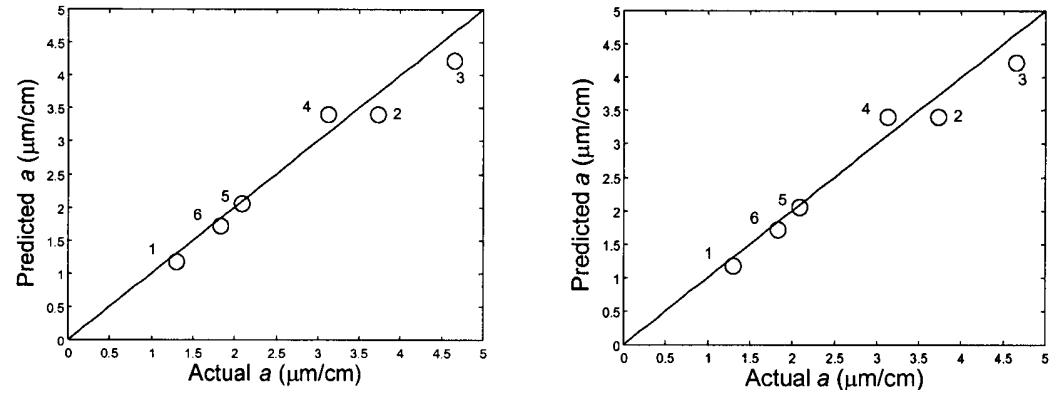

Fig. 11. Measured and predicted values of ANFIS output.

Table 2. Overall performance of the ANFIS implementation in predicting $\delta_{\text {agg }}$.

\begin{tabular}{llll}
\hline Test & Max. error $(\mu \mathrm{m})$ & Min. error $(\mu \mathrm{m})$ & Mean error $(\mu \mathrm{m})$ \\
\hline 1 & 3.0041 & 0.2215 & 1.4924 \\
2 & 6.1654 & 0.2198 & 3.4490 \\
3 & 8.7150 & 0.7995 & 3.1251 \\
4 & 5.2113 & 0.6443 & 2.7839 \\
5 & 2.1174 & 0.2666 & 1.2180 \\
6 & 3.4730 & 0.2185 & 1.4887 \\
\hline
\end{tabular}

designed to benefit from the well-developed state of the art in the cutting force prediction in turning operations, thus simplifying the task of learning. However, further work is required before the new ANN approach could be translated to other cutting operations such as end milling.

The model-based error compensation approach developed in $[2,3]$ had sought to use case-based reasoning (CBR) to effect learning over the variety of cutting operations usually experienced by the machine. However, learning through CBR is essentially fragmented, and intermittent. Further, CBR does not exploit useful information that might be obtained from past experience that was dissimilar to the new cutting situation (for which the total error must be predicted). A similar problem exists with the off-line training required by the hybrid ANN system used in the present paper. However, it seems that this is not a fundamental limitation of ANN, but only of its current state of development. This optimism arises from the fact that recent literature on ANN describes several new ANN architectures that enable continuous learning. As these developments mature, the learning system described in the present paper may be progressively modified so that, eventually, we could realise the vision of achieving a system that enables a CNC machine to predict machining errors autonomously solely on the basis of normal shop-floor activities (machining and inspection) being undertaken by it. It is believed that the work reported in this paper represents a small but significant step in this direction.

\section{Acknowledgement}

This paper has been made possible by the funding (CERG 9040308) received from the Research Grants Council of Hong Kong. The authors are grateful to the Council for the fund. The authors also thank Professor V. A. Ostafiev of Kiev 
Polytechnic, Ukraine, for making available the "fine touch" sensor used in the on-machine measurements. Further, the contributions made by Victor Trush and He Yu-zhu, Research Assistants, to the development of soft touch are gratefully acknowledged.

\section{References}

1. J. Bryan, "International status of thermal error research," Annals CIRP, 39(2), pp. 645-656, 1990.

2. Z. Q. Liu and P. K. Venuvinod, "Error compensation in CNC turning solely from dimensional measurements of previously machined parts," Annals CIRP, 48(1), pp. 429-432, 1999.

3. Z. Q. Liu, "Error compensation in CNC turning from dimensional measurements of previously machined parts," $\mathrm{PhD}$ thesis, City University of Hong Kong, 1999.

4. V. A. Ostafiev and P. K. Venuvinod, "A new electro-magnetic contact sensing technique for enhancing machining accuracy," International Journal of Manufacturing Science and Technology, ASME, MED-6 (1), pp. 113-119, 1997.

5. Z. Q. Liu P. K. Venuvinod and V. A. Ostafiev, "On-machine measurements of workpieces with cutting tool," International Journal of Manufacturing Technology Management, 9(3), pp. 168172,1998

6. S. K. Birla, "Sensors for adaptive control and machine diagnostics," Proceedings Machine Tool Task Force Conference 4, pp. $7-12,1980$.

7. V. A. Ostafiev and A. Djordjevich, "Machining precision augmented by sensors," International Journal of Production Research, 37, pp. 91-98, 1999.
8. J. Mou and C. R. Liu, "An error correction method for CNC machine tools using reference parts," Transactions of NAMRI/SME, 22, pp. 275-282, 1994.

9. C. A. van Luttervelt, T. H. C. Childs, F. Klocke and P. K. Venuvinod, "Present situation and future trends in modelling of machining operations, Progress Report of the CIRP working group 'Modelling of Machining Operations'", Annals CIRP, 47 (2) pp. 587-626, 1998.

10. S. Yang, J. Yuan and J. Ni, "Real-time cutting force induced error compensation on a turning center," International Journal of Machine Tools and Manufacture, 37, pp. 1579-1610, 1997.

11. P. K. Venuvinod, "Prediction augmentation through reinforcing interactions amongst modeling, sensing and learning, Session on 'Modeling of Machining Operations, STC Cutting' " CIRP General Assembly, Part II, Tianjin, China, 1997.

12. P. K. Venuvinod, "Sensor-based modeling of machining operations," CIRP International Workshop on Modeling of Machining Operations, Atlanta, USA, 1998.

13. P. K. Venuvinod and W. L. Jin, "Three dimensional cutting force analysis based on the lower boundary of shear zone: Part 1: Single edge oblique cutting," International Journal of Machine Tools and Manufacture, 36 (3), pp. 307-323, 1996.

14. R. Azouzi and M. Guillot, "On-line prediction of surface finish and dimensional deviation in turning using neural network based sensor fusion," International Journal of Machine Tools and Manufacture, 37, pp. 1201-1217, 1997.

15. T. S. Suneel, H. L. Shashidhara, S. S. Pande and V. M. Gadre, "Simulation studies using wavelet neural networks for intelligent CNC turning," Proceedings of the International Conference on Artificial Intelligence, Las Vegas, Nevada, USA, pp. 231-238, 1999.

16. R. Teti and S. R. T. Kumara, "Intelligent computing methods for manufacturing systems," Annals CIRP, 46 (2), pp. 629-652, 1997.

17. J. S. R. Jang, "Adaptive network based fuzzy inference systems," IEEE Transactions on Systems, Man, and Cybernetics, 23 (3), pp. 665-685, 1993. 